\title{
VLA and ATCA observations of the nuclear activity in selected southern Seyfert galaxies
}

\author{
Rosane Bohrer-Adornes ${ }^{1}$ and Everton Lüdke ${ }^{1}$ \\ ${ }^{1}$ Universidade Federal de Santa Maria, CCNE - Departamento de Física - LARIE, Campus \\ Universitário, 97150-900 Santa Maria RS, Brazil
}

\begin{abstract}
This work reports our recent results of an HI line and radio continuum imaging survey of selected southern Active galaxies made with the Very Large Array and the Australian Telescope Compact Array. Radio images are presented and rotation curves for individual galaxies were obtained. The HI total mass content in Seyfert galaxies is larger than those seen in starburst galaxies. Our trends confirm that the infrared luminosity correlates well with the total neutral gas content, which supports the scenario that star formation in the bulges of Seyfert galaxies is considerably higher than in regular late-type galaxies or spirals. We also find a trend between various observational quantities which are also discussed in this work.
\end{abstract}

\section{Introduction}

The study of the circumnuclear regions in spiral galaxies with optically-identified Seyfert nuclei has proved to be a very important tool for the understanding of the origin of the optical and radio activity in spiral galaxies (Osterbrock 1991). In the so-called Seyfert galaxies, nonthermal radio emission originates in the galactic disk and is enhanced towards the optical cores (Phillips et al. 1983). Massive black hole formation (Blandford 1992) and violent star formation (Sanders \& Bania 1976) have been invoked to explain the optical variability and the luminosity of these structures. In fact, observations of archetypical starburst galaxies with Very Long Baseline Techniques have shown evidence of an Active nuclei (Smith et al. 1998) associated to compact radio cores. X-ray and mid-infrared direct imaging are also helping to identify hidden AGNs in selected starbursts.

Modern radio interferometry techniques with modest baselines (5 12 km) are an useful tool to obtain images of the relativistic particle distribution in the interstellar medium and circumnuclear regions of AGNs, from which various diagnostics regarding the origin of activity in spiral and irregular galaxies can be investigated. In this paper, we present preliminary results concerning a survey of $z<0.008$ Seyfert galaxies in the southern hemisphere and observational properties of individual galaxies are also given.

\section{Discussion}

The Australia Telescope Compact Array have been used to produce radio continuum maps of eleven southern Seyfert galaxies to study the radio emission properties at 1380 MHz. The selected objects are NGC1566, NGC1672, NGC3109, NGC4945, NGC5068, NGC6221, NGC6684, NGC6744, IC5201, NGC7314 and NGC7424. Physical parameters are calculated from the radio continuum images and are shown in table 1.

Radio structures were detected within a field radius of $\sim 1.4 \mathrm{kpc}$ from the optical cores and are partially resolved in our present images. Nuclear magnetic fields range from 30 to $160 \mu \mathrm{G}$ from minimum energy analysis. The magnetic fields confined to 
Table 1. Derived properties from radio continuum images at $1384 \mathrm{MHz}$, from the guidelines given by Condon (1992).

\begin{tabular}{lllllllll}
\hline Name & $\begin{array}{c}\mathrm{D} \\
(\mathrm{Mpc})\end{array}$ & $\begin{array}{l}\log P_{n} \\
\left(\mathrm{~W} \mathrm{~Hz}{ }^{-1}\right)\end{array}$ & $\begin{array}{l}\log L_{n} \\
(\mathrm{~W})\end{array}$ & $\begin{array}{l}\log L_{F I R} \\
\left(L_{\odot}\right)\end{array}$ & $\begin{array}{l}\mathrm{Tb} \\
(\mathrm{K})\end{array}$ & $\begin{array}{l}B_{e q} \\
(\mu \mathrm{G})\end{array}$ & $\begin{array}{l}\log U_{m} \\
(\mathrm{ergs})\end{array}$ & $\begin{array}{l}\mathrm{SFR} \\
\mathrm{M}_{\odot} \mathrm{yr}^{-1}\end{array}$ \\
\hline NGC1566 & 20.0 & 21.88 & 31.24 & 10.11 & 760 & 45 & 59.45 & 1.82 \\
NGC1672 & 18.0 & 22.31 & 32.20 & 10.28 & 2297 & 62 & 59.68 & 4.94 \\
NGC3109 & 5.4 & 20.10 & 28.81 & 8.27 & 157 & 41 & 57.74 & 0.03 \\
NGC4945 & 7.5 & 22.39 & 33.12 & 10.52 & 18820 & 158 & 59.14 & 5.91 \\
NGC5068 & 9.0 & 20.46 & 29.10 & 9.03 & 132 & 34 & 58.22 & 0.07 \\
NGC6221 & 19.8 & 22.03 & 31.54 & 9.49 & 1360 & 55 & 59.47 & 2.56 \\
NGC6684 & 11.8 & 21.08 & 30.10 & $<8.2$ & 296 & 39 & 58.74 & 0.29 \\
NGC6744 & 11.2 & 20.67 & 29.32 & 8.44 & 218 & 36 & 58.40 & 0.11 \\
IC5201 & 12.2 & 21.30 & 30.50 & 8.71 & 594 & 46 & 58.86 & 0.48 \\
NGC7314 & 19.0 & 21.60 & 30.73 & 9.51 & 311 & 33 & 59.38 & 0.97 \\
NGC7424 & 12.6 & 21.39 & 30.66 & 8.82 & 488 & 46 & 58.95 & 0.59 \\
\hline
\end{tabular}

the cores are at least about 30 times higher than those in the disks of normal spiral galaxies. Simple star-formation models suggest that the circumnuclear radio emission can be explained by massive star formation, and star formation rates have been calculated for individual objects ranging from normal disk values $\left(\sim 0.003 \mathrm{M}_{\odot} \mathrm{yr}^{-1}\right)$ to extremely high values $\left(\sim 6.0 \mathrm{M}_{\odot} \mathrm{yr}^{-1}\right)$. NGC1672, NGC4945 and NGC6221 require very high star formation rates $\left(\mathrm{SFR}>2 \mathrm{M}_{\odot} \mathrm{yr}^{-1}\right.$ ) to explain the observed radio continuum, whereas the remaining galaxies show modest values for the star-formation rates. We confirm a correlation between the nuclear radio power and far-infrared luminosities for our sample galaxies, similar to those established for normal galaxies by various authors (Roy \& Norris 1997; Roy et al. 1998).

\section{Acknowledgements}

FAPERGS, CAPES and CNPq/Brazilian Government are acknowledged for research. E. Lüdke would like to thank both ATCA and NRAO staff for technical support. The VLA is operated by the NRAO under contract with the NSF. The ATCA is operated by the ATNF/CSIRO on behalf of the Commonwealth.

\section{References}

Blandford, R. D. 1992, in Physics of Active Galactic Nuclei, (eds. W.J. Duschl \& S.J. Wagner), Springer-Verlag, p.3

Condon, J. J. 1992, ARA\&A, 30, 575

Osterbrock, D. E. 1991, Rep. Prog. Phys., 54, 579

Phillips, M. M., Charles, P. A., \& Baldwin, J. A. 1983, ApJ, 266, 485

Roy A. L., \& Norris, R. P. 1997, MNRAS, 289, 824

Roy, A. L., Norris, R. P., Kesteven, M. J., Troup, E. R., \& Reynolds, J. E. 1998, MNRAS, 301, 1019

Sanders, R. H., \& Bania, T. M. 1976, ApJ, 204, 341

Smith, H. E., Lonsdale, C. J., \& Lonsdale, C. J. 1998, ApJ, 492, 137 